\title{
Study on the Antianxiety Mechanism of Suanzaoren Decoction Based on Network Pharmacology and Molecular Docking
}

\author{
Xiaocong Xu $\mathbb{D},{ }^{1}$ Bingbing Gao $\mathbb{D},^{2}$ Xiongying $\mathrm{Li}\left(\mathbb{D},{ }^{3}\right.$ and Shanshan Lei $\mathbb{D}^{4}$ \\ ${ }^{1}$ Zhejiang Chinese Medical University, Hangzhou, Zhejiang 310015, China \\ ${ }^{2}$ The Second Affiliated Hospital of Zhejiang Chinese Medical University, Hangzhou, Zhejiang 310015, China \\ ${ }^{3}$ Jiangxi College of Traditional Chinese Medicine, Fuzhou, Jiangxi 344000, China \\ ${ }^{4}$ Department of Medicine, Zhejiang Academy of Traditional Chinese Medicine, Hangzhou, Zhejiang 310015, China \\ Correspondence should be addressed to Xiongying Li; 344504439@qq.com and Shanshan Lei; leishan199006@126.com
}

Received 23 February 2021; Revised 18 May 2021; Accepted 1 July 2021; Published 31 July 2021

Academic Editor: Caio P. Fernandes

Copyright (๑) 2021 Xiaocong Xu et al. This is an open access article distributed under the Creative Commons Attribution License, which permits unrestricted use, distribution, and reproduction in any medium, provided the original work is properly cited.

\begin{abstract}
Objective. Suanzaoren Decoction (SZRT) is a classic decoction to calm the nerves in traditional Chinese medicine (TCM). It has been extensively treated as an antianxiety drug in modern times, but the material basis and pharmacological mechanisms are still unclear. To explore the material basis and corresponding potential targets, as well as to elucidate the mechanism of SZRT, network pharmacology and molecular docking methods were utilized. Methods. The main chemical compounds and potential targets of SZRT were collected from the pharmacological database analysis platform (TCMSP). Anxiety targets were obtained from the GeneCards database. Then, a target compound network was established using overlapping genes and the corresponding potential compounds. Protein interaction analysis, GO enrichment, and KEGG pathway enrichment were performed using the STRING database, DAVID database, and KOBAS database. Finally, molecular docking was conducted between MAOB and its corresponding active compound in SZRT to further verify the results. Results. A total of 137 active components in SZRT were screened from the TCMSP database, and 210 corresponding targets were predicted. A total of 5434 anxietyrelated targets were obtained from the disease target database, and finally 22 potential targets of SZRT on antianxiety were obtained. The constructed C-T network showed that the average degree of active components was 5.4, and four of them interacted with six or more targets. PPI analysis shows that key genes such as MAOA, MAOB, IL1B, TNF, NR3CI, and HTR3A were identified as potential therapeutic targets. A pathway analysis showed that SZRT may participate in neurotransmitter regulation and immunoregulation in a synergistic way to treat anxiety. The binding energy between the active compounds and MAOB was low, indicating good binding. The results of molecular docking showed that all the 10 active ingredients were able to successfully dock with MAOB, and the binding energy of coumaroyltyramine with MAOB was the lowest, that is, $-9.6 \mathrm{kcal} /$ $\mathrm{mol}$, and the binding method was hydrogen bonding. Conclusions. SZRT produces antianxiety effects mainly by affecting the neurotransmitter release, transmission, and immunoregulation. This study provides a new approach to elucidating the molecular mechanism and material basis of SZRT in the treatment of anxiety, and it will also benefit the application of TCM in modern medicine.
\end{abstract}

\section{Introduction}

Anxiety disorder is the most common type of mental illness. It is manifested by persistent anxiety, tension, panic, and other anxieties, accompanied by autonomic disorders, muscle tension, and kinesthesia. Most of the symptoms start early in children, adolescents, and adults. Unlike normal or stress-induced transient anxiety, it is continuous and impairs daily functions. Women's anxiety is twice as much as men. Anxiety disorder mainly includes generalized anxiety disorder (GAD) and panic disorder (PD). GAD, also known as "chronic anxiety," is characterized by excessive anxiety, which is generalized and persistent, often exhibiting somatic symptoms, including headache and gastrointestinal symptoms [1]. PD, also known as "acute anxiety," is a type of acute recurrent episodes of severe anxiety (panic) 
characterized by sudden high levels of fear (often reaching a peak within a few minutes [2]) which may be accompanied by palpitations and difficulty in breathing, profuse sweating, dizziness, general numbness, and other physical symptoms.

The pathogenesis of anxiety disorders is complex, and it has not been elucidated yet. The molecular mechanism is related to neurotransmitters and their receptors, hypothalamus-pituitary-adrenal axis, hypothalamus-pituitaryadrenal axis, opioid nervous system, hormone levels, oxidation, etc. Many drugs are available to treat anxiety, including adrenergic receptor blockers (e.g., propranolol), tricyclic antidepressants (TCA and imipramine), monoamine oxidase inhibitors (MAOIs such as phenylethyl trap), and benzodiazepines (e.g., DZP), selective 5-HT reuptake inhibitors (e.g., fluoxetine), selective 5-HT receptor ligand inhibitors (e.g., buspirone), and HT1A receptor partial agonists. However, these drugs have side effects partly, such as anticholinergic cardiovascular, autonomic dysfunction, or drug dependence [3].

Traditional Chinese medicine (TCM) has been used in China for thousands of years and exhibits good therapeutic effects, few side effects, and multiple pathway regulation for the prevention and treatment of anxiety. Suanzaoren Decoction (SZRT) first recorded in "Shennong Bencao Jing" consists of five herbs with different percentages in weight: Ziziphi Spinosae Semen (SZR, 33.33\%), Glycyrrhizae Radix et Rhizoma (GC, 13.33\%), Anemarrhenae Rhizoma (ZM, 13.33\%), Poria (FL, 26.67\%), and Chuanxiong Rhizoma (CX, 13.33\%). It has exhibited excellent effect on antianxiety or anti-insomnia in clinical practice in China for hundreds of years. Modern clinical studies showed that SZRT could improve GAD [4] and anxiety insomnia [5]; pharmacological studies also validated that SZRT can effectively improve anxiety disorder in a high plus maze experiment of rats and clinically alleviate anxiety symptoms [6]. The alcohol extracts of Ziziphi Spinosae Semen [7] and Anemarrhenae Rhizoma [8] had an antianxiety-like behavior in mice. Although the therapeutic effect of SZRT is attractive, the molecular mechanism of action has not been well understood. Although some traditional approaches including chemical analyses [7] and bioactivity tests have been performed to study SZRT, its pharmacological mechanism and material basis have not been fully elucidated.

Here, based on the system's pharmacology framework [9], it was used to evaluated the molecular pathogenesis of anxiety and elucidate the action mechanism of clinically effective formula SZRT. First, the active ingredients of SZRT were screened via OB (oral bioavailability) and DL (drug-likeness) evaluation. Then, the active ingredients were utilized as baits to predict the potential targets, and the compound-target interactions at the pharmacological level were determined. Subsequently, the targets were used as baits to determine the corresponding pathways from the KEGG database [10]. A target-pathway (T-P) network was constructed for network analysis. The results will be helpful for understanding of anxiety pathogenesis, but also clarified the molecular mechanism of SZRT, promoting the development of TCM in the treatment of anxiety. An overview of the experimental procedures for SZRT is shown in Figure 1.

\section{Methods}

2.1. Screening of Active Ingredients. The TCM System Pharmacology Database (TCMSP, http://lsp.nwu.edu.cn/ tcmsp.php) [11] is generally used as a platform for systems pharmacology and a compound ingredient database. In this study, the TCMSP database was used to search the ingredients of SZRT. Ziziphi Spinosae Semen (Suan Zao Ren, SZR), Glycyrrhizae Radix et Rhizoma (Gan Cao, GC), Anemarrhenae Rhizoma (Zhi Mu, ZM), Poria (Fu Lin, FL), and Chuanxiong Rhizoma (Chuan Xiong, CX) were entered into the retrieval field, and the ingredients of each herb and ADME characteristics were obtained. Among the ADME characteristics, the threshold values of $\mathrm{OB}$ were set as $\geq 30 \%$, and DL was set as $\geq 0.18$. Finally, the matched ingredients were treated as the candidate active ingredients.

2.2. Collection of Anxiety-Related Targets and Construction of Active Ingredients-Target Network. The candidate ingredients of five herbs (including SZR, GC, ZM, FL, and CX) of SZRT were put into the TCMSP database, and the candidate protein targets were acquired. Then, we collected and arranged these targets using Excel and finally received the candidate protein targets corresponding to SZRT. Then, the UniProt Knowledgebase (http://www.uniprot.org/) was used to convert the screened-out protein names to gene names. In the end, the dataset of SZRT active compounds and related target genes were obtained.

Additionally, GeneCards (https://www.genecards.org/) was used to collect anxiety-related target genes, and the term anxiety was searched as the keyword. Then, the dataset of SZRT compounds and related target genes and genes related to anxiety were integrated, and the overlapping genes were determined as the SZRT's active compounds' target genes on antianxiety. Cytoscape 3.2.1 software was used to establish the active compounds-anxiety target genes network (C-T network).

2.3. PPI and Hub Gene Analysis. Import the screened-out target genes into the STRING database (https://string-db. org/) for analyzing the protein interaction and establishing the PPI network diagram. The PPI data were imported into Cytoscape 3.2.1 software for visual analysis, and cytoHubba plug-in was used to find the hub gene mapping from SZRT to the onset of anxiety.

2.4. GO Enrichment Analysis. The intersection targets of SZRT and anxiety were imported into the DAVID (https:// david.ncifcrf.gov/) database for GO function enrichment analysis. GO function annotations mainly include biological process (BP), cell component (CC), and molecular function (MF). Enriched GO terms were defined as significant with $P<0.05$, and the top 10 enriched functions were analyzed and drawn using GraphPad Prism 8.0 software. 


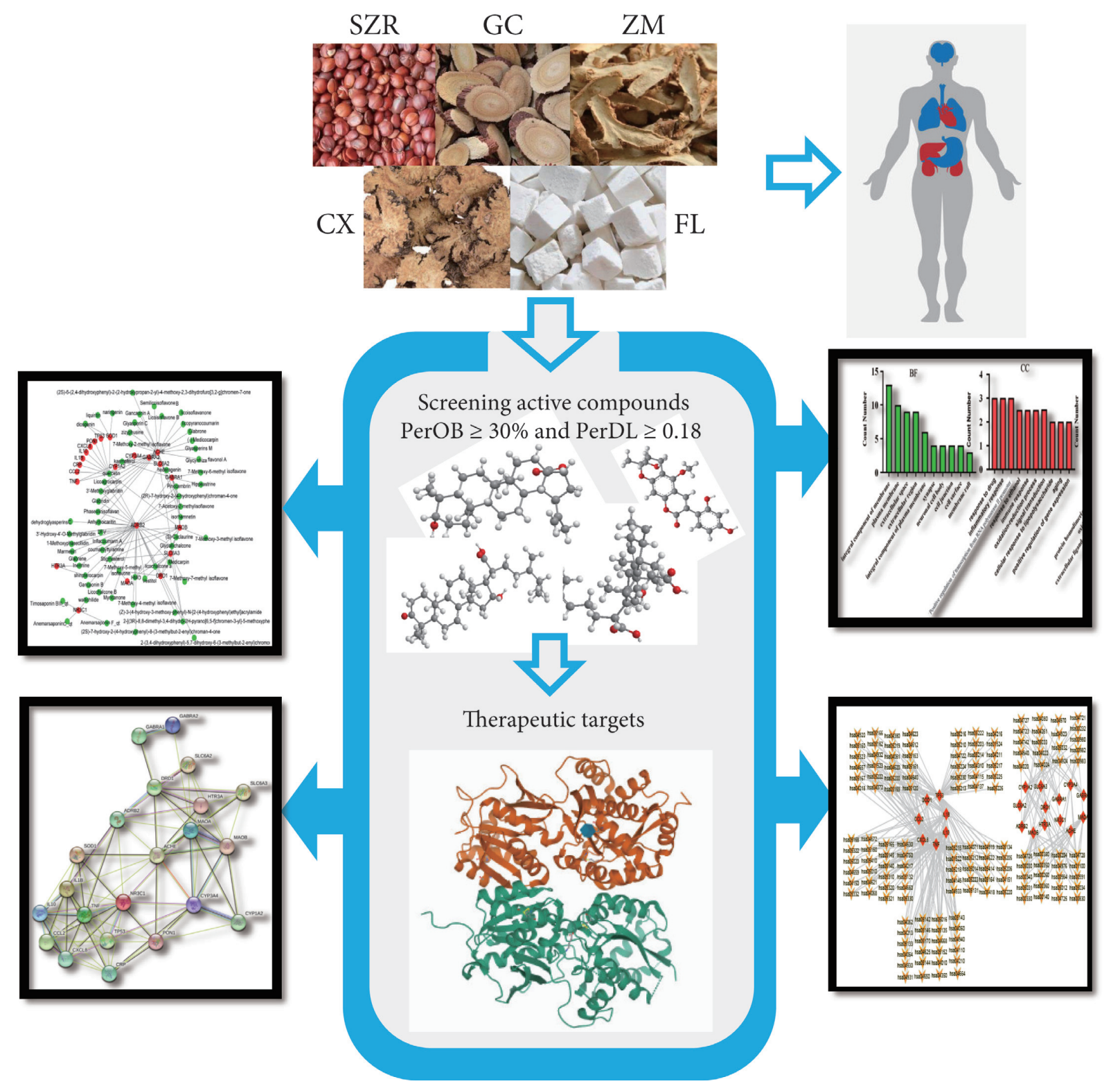

FIgURE 1: An overview of the experimental procedures for SZRT.

2.5. Establishment of T-P Network. The DAVID (https://david. ncifcrf.gov/) database was used to transform the target genes' ID; then, the KOBAS database (http://kobas.cbi.pku.edu.cn/) was utilized for KEGG pathway enrichment analysis. Cytoscape software 3.2.1 was used to establish a visualized network of T-P network. In the networks, each target or pathway was expressed by one node, and each interaction was represented by an edge.

2.6. Autodocking of Active Compounds of SZRT with Target Protein. AutoDock 4.2.6 was used (http://autodock. Scripps.Edu/news/autodock4-2.6) as a molecular docking software for flexible docking. Analysis of KEGG pathways showed that MAOB is a vital protein target. The structure of MAOB was obtained from the PDB database (http://www.rcsb.org/pdb/home/home.do); the protein code was PDB 2V60. Using PyMOL 2.7 software, the primary ligand conformation of the target protein was extracted and saved as a PDB format file. Then, AutoDock tools (ADT) was used to remove the water molecules. Hydrogenation and charge treatment of MAOB were conducted and saved as a PDBQT format file. The ligands of Mol2 format file were processed by ADT and saved as a PDBQT format. Then, the docking results of compounds and proteins were analyzed using AutoDock and visualized using PyMOL 2.7.

\section{Results}

3.1. Active Compounds of SZRT. From the TCMSP database, a total of 617 related compounds in the SZRT were found: 33 ingredients (5.3\%) in Suan Zao Ren (Ziziphi Spinosae Semen, SZR), 280 ingredients (45.4\%) in Gan Cao (Glycyrrhizae 
Radix et Rhizoma, GC), 81 ingredients (13.1\%) in Zhi Mu (Anemarrhenae Rhizoma, ZM), 34 ingredients (5.5\%) in Fu Lin (Poria, FL), and 189 ingredients (30.6\%) in Chuan Xiong (Chuanxiong Rhizoma, CX). To screen out the active ingredients, the ADME properties ( $\mathrm{OB} \geq 30 \%, \mathrm{DL} \geq 0.18$ ) were used to evaluate these ingredients. As a result, 137 active compounds were obtained. Then, the herb-active compounds were constructed as shown in Table 1.

For 137 ingredients, many of them were validated to display vital biological activities including immunoregulatory activities, neurotransmitter regulation, and endocrine regulation. For instance, swertisin with good pharmacokinetic profiles was obtained from SZR, with multifarious pharmacological properties including neurotransmitter regulation activity and adenosine A1 receptor (ADORA1) antagonistic property [12]. Kaempferol markedly protected against CPF-induced neuronal damage, increased the AChE activity, and induced a better performance in the behavioral tests [13]. These results indicate that the active compounds may show synergetic pharmacological effects on anxiety. Besides, mairin is a common potential active ingredient of SZR and GC; sitosterol is a common potential active ingredient of GC and CX; and kaempferol is a common potential active ingredient of ZM and GC. These candidate compounds could be the key elements for curing anxiety.

3.2. Compound-Targets (C-T) Network Analysis. The compounds which had no targets based on the TCMSP database or did not have the corresponding gene name in the UniProt database were deleted; finally, 205 target genes corresponding to 112 compounds were obtained. The pathogenesis of anxiety is very complex. A total of 5434 anxietyrelated genes were found in the GeneCards database, and 336 anxiety-related targets with a relevance score of $\geq 10$ and $P<0.05$ were obtained. To determine which of the 205 target genes are related to anxiety, the target genes were further mapped to 336 anxiety-related targets, and finally 22 overlapping target genes were screened out. A visual graph of the C-T network was established based on the potential ingredients and targets shown in Figure 2. The C-T network had a total of 79 nodes (22 targets and 57 active components), forming 119 relationships. The C-T network analysis shows that the average degree of per target to compound is 5.4, elucidating the multiple target properties of SZRT.

The C-T network analysis showed that four active ingredients could interact with six or more targets, including quercetin, kaempferol, stigmasterol, and 7-methoxy-2methyl isoflavone. Moreover, seven targets could interact with more than four active ingredients, including acetylcholinesterase (ACHE), beta-2 adrenergic receptor (ADRB2), gamma-aminobutyric acid receptor subunit alpha-1 (GABRA1), amine oxidase (flavin-containing) B (MAOB), glucocorticoid receptor (NR3C1), sodium-dependent dopamine transporter (SLC6A3), and superoxide dismutase $[\mathrm{Cu}-\mathrm{Zn}]$ (SOD1), indicating the antianxiety effect of SZRT with multiple pathways and multiple targets. The results are shown in Table 2.
3.3. PPI and Hub Gene Analysis. The data obtained from the STRING database were loaded to Cytoscape software, and 22 target genes were found to form a total of 84 protein interaction relationships as shown in Figure 3. The combined score of 20 of the protein interaction relationships was $\geq 0.9$, which was regarded as a high degree of confidence. The protein interaction network was then imported into Cytoscape software 3.2.1 and analyzed using cytoHubba plug-in; the top 10 key genes were obtained according to the degree. Among these hub target genes, CRP, TP53, IL1B, TNF, and CCL2 are inflammatory factors. 5-HT is a vital neurotransmitter in the control of anxiety. HTR3A is a 5-HT receptor; MAOA resolves 5-HT. These indicate that SZRT antianxiety might regulate inflammatory factors and neurotransmitters.

3.4. GO Enrichment Analysis. A GO enrichment analysis was performed on the 22 potential targets obtained, sorting the BP terms, cellular component (CC), and MF terms in the ascending order of $\log P$ value. The top 10 are shown in Figure 4. As shown in Figure 4, the BPs were mainly involved in inflammatory response, immune response, redox process, and signal transduction. The CCs were mainly involved in the integral components of membrane, plasma membrane, extracellular space, extracellular region, integral component of plasma membrane, synapse, neuronal cell body, cell junction, cell surface, and membrane raft. The MFs were mainly involved in oxidoreductase activity, extracellular ligand-gated ion channel activity, cytokine activity, enzyme binding, dopamine:sodium symporter activity, caffeine oxidase activity, GABA-gated chloride ion channel activity, and primary amine oxidase activity. All these results indicate that SZRT could be used for the treatment of antianxiety by multiple pathways and exhibits a synergistic effect by interactive effect.

\subsection{KEGG Pathway Enrichment Analysis and T-P Network} Analysis. As two of the 22 target genes did not map into the pathways in the KOBAS database, 20 targets were mapped to 149 pathways. The T-P network including 169 nodes and 303 edges is shown in Figure 5. In the T-P network, the average degree per target gene was 15.1. We also found that 16 targets could be mapped into multiple pathways $(n \geq 5)$, which might be the vital factors for anxiety. Additionally, 39 pathways were also interceded by a variety of targets $(n \geq 3)$, indicating that cross-talk between multiple pathways and targets might be the highlight of pathogenesis of anxiety.

To further find the key pathways, the top 40 pathways were analyzed; 16 pathways were related to anxiety as shown in Table 2. NF-kappa B signaling pathway, cytokine-cytokine receptor interaction, IL-17 signaling pathway, toll-like receptor signaling pathway, TNF signaling pathway, NOD-like receptor signaling pathway, and C-type lectin receptor signaling pathway belong to immunoregulation. Dopaminergic synapse, serotonergic synapse, tryptophan metabolism, amphetamine addiction, phenylalanine metabolism, and neuroactive ligand-receptor interaction belong to multineurotransmitter regulation. 
TABLE 1: The main active material parameters of SZRT.

\begin{tabular}{|c|c|c|c|c|}
\hline Herb & Molecule name & $\begin{array}{l}\text { OB } \\
(\%)\end{array}$ & $\mathrm{DL}$ & Structure \\
\hline GC & DFV & 32.76 & 0.18 & \\
\hline GC & Glycyrrhiza flavonol A & 41.28 & 0.6 & \\
\hline GC & Pinocembrin & 64.72 & 0.18 & \\
\hline GC & 1-Methoxyphaseollidin & 69.98 & 0.64 & \\
\hline GC & Glypallichalcone & 61.6 & 0.19 & \\
\hline GC & Licopyranocoumarin & 80.36 & 0.65 & \\
\hline GC & Licochalcone B & 76.76 & 0.19 & \\
\hline GC & Glycyrol & 90.78 & 0.67 & \\
\hline
\end{tabular}


TABle 1: Continued.

\begin{tabular}{|c|c|c|c|c|}
\hline Herb & Molecule name & $\begin{array}{l}\text { OB } \\
(\%)\end{array}$ & $\mathrm{DL}$ & Structure \\
\hline GC & Icos-5-enoic acid & 30.7 & 0.2 & \\
\hline GC & $18 \alpha$-Hydroxyglycyrrhetic acid & 41.16 & 0.71 & \\
\hline GC & Gadelaidic acid & 30.7 & 0.2 & \\
\hline GC & Shinpterocarpin & 80.3 & 0.73 & \\
\hline
\end{tabular}


TABle 1: Continued.

Herb Molecule name Structure

GC,

CX

GC

GC

GC

GC
Sitosterol

HMO

Gancaonin H

Vestitol

Glycyroside

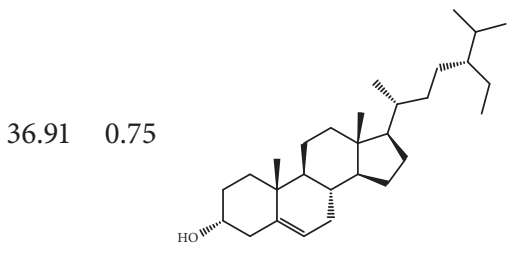

38.37<smiles>COc1ccc2c(=O)c(-c3ccc(Br)cc3)coc2c1</smiles>

50.1<smiles>CC(CCc1c(O)cc2occ(-c3cc(N)c4c(c3)CCC(C)(C)O4)c(=O)c2c1Cl)=[Hg]O</smiles>

74.66

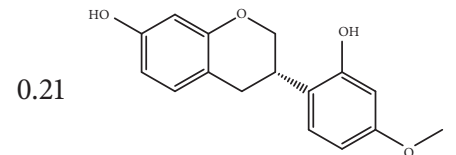

37.25

0.79

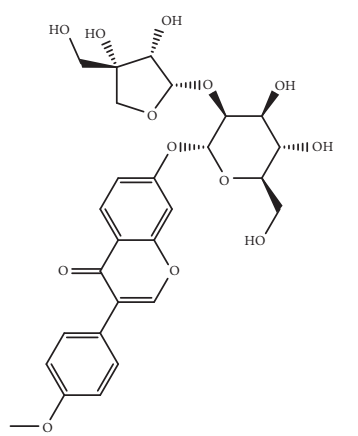


TABle 1: Continued.

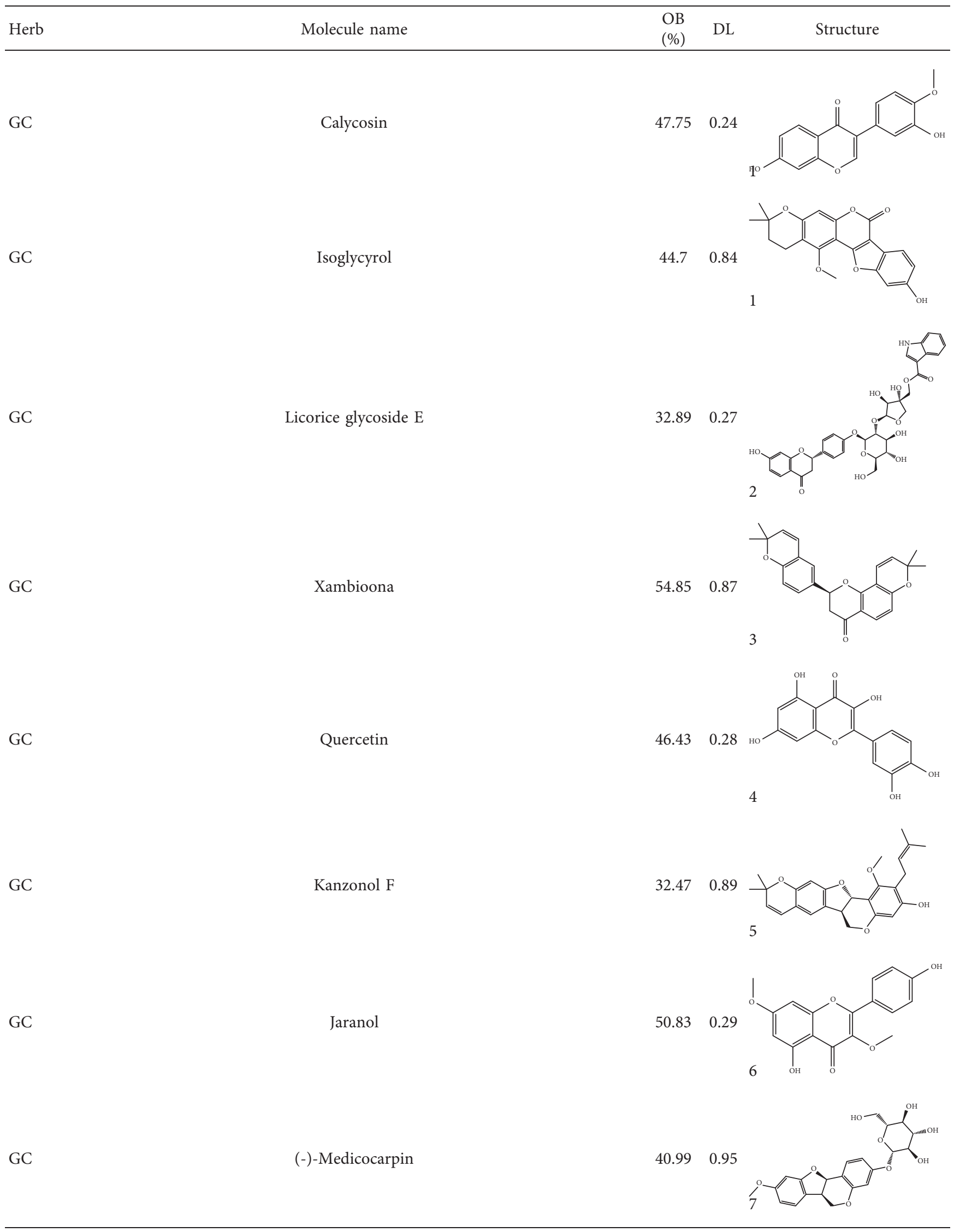


TABle 1: Continued.

\begin{tabular}{|c|c|c|c|c|}
\hline Herb & Molecule name & $\begin{array}{l}\mathrm{OB} \\
(\%)\end{array}$ & $\mathrm{DL}$ & Structure \\
\hline
\end{tabular}

GC

GC

GC

ZM

GC

$\mathrm{ZM}$

GC

$\mathrm{ZM}$,

GC
Licochalcone a

Euchrenone

Odoratin

Marmesin

Isorhamnetin

Coumaroyltyramine

Glabranin

Kaempferol
40.79<smiles>C=CC(C)(C)c1cc(/C=C/C(=O)c2ccc([N+](=O)[O-])cc2)c(OC)cc1O</smiles>

30.29<smiles>C/C=C\C1=C(OC(C)C)C=CC(C2CC(=O)c3ccc(O)c(CC=C(C)C[SeH3+])c3O2)C1</smiles>

49.95

0.3<smiles>COc1cc2c(=O)c(-c3ccc(OC)c(Cl)c3)coc2cc1O</smiles>

50.28<smiles>CC(C)(C)C1Cc2cc3ccc(=O)oc3cc2O1</smiles>

49.6<smiles>CC(C)=CCc1cc(-c2oc3cc(O)cc(O)c3c(=O)c2O)ccc1C</smiles>

112.9<smiles>O=C(/C=C\c1ccc(Cl)cc1)NCCc1ccc(O)cc1</smiles>

$52.9 \quad 0.31$<smiles>CC1(C)C=Cc2c(ccc3c2OC[C@@H](c2ccc(O)cc2O)C3)O1</smiles>

41.88<smiles></smiles> 
TABle 1: Continued.

\begin{tabular}{|c|c|c|c|c|}
\hline Herb & Molecule name & $\begin{array}{l}\text { OB } \\
(\%)\end{array}$ & $\mathrm{DL}$ & Structure \\
\hline GC & Licochalcone G & 49.25 & 0.32 & \\
\hline $\mathrm{ZM}$ & Anhydroicaritin & 45.41 & 0.44 & \\
\hline GC & Quercetin der. & 46.45 & 0.33 & \\
\hline $\mathrm{ZM}$ & Asperglaucide & 58.02 & 0.52 & \\
\hline GC & Inflacoumarin $\mathrm{A}$ & 39.71 & 0.33 & \\
\hline $\mathrm{ZM}$ & Chrysanthemaxanthin & 38.72 & 0.58 & \\
\hline
\end{tabular}


TABle 1: Continued.

\begin{tabular}{|c|c|c|c|c|}
\hline Herb & Molecule name & $\begin{array}{l}\text { OB } \\
(\%)\end{array}$ & DL & Structure \\
\hline
\end{tabular}

GC

$\mathrm{ZM}$

GC

$\mathrm{ZM}$

GC

$\mathrm{ZM}$

GC

Glyzaglabrin

Medicarpin

Icariin I

Stigmasterol
Glepidotin B

Hippeastrine

Glepidotin A

43.83

0.76

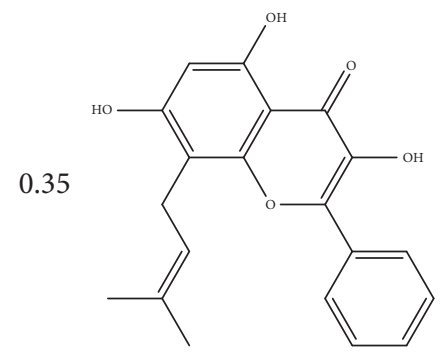

$44.72 \quad 0.35$

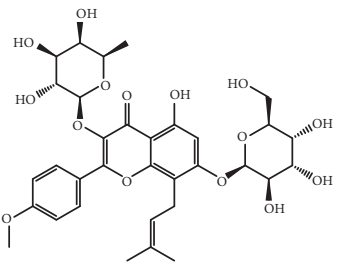<smiles>CC(C)=CCc1c(O)cc(O)c2c1O[C@H](c1ccccc1)[C@H](C)C2=O</smiles>

51.65
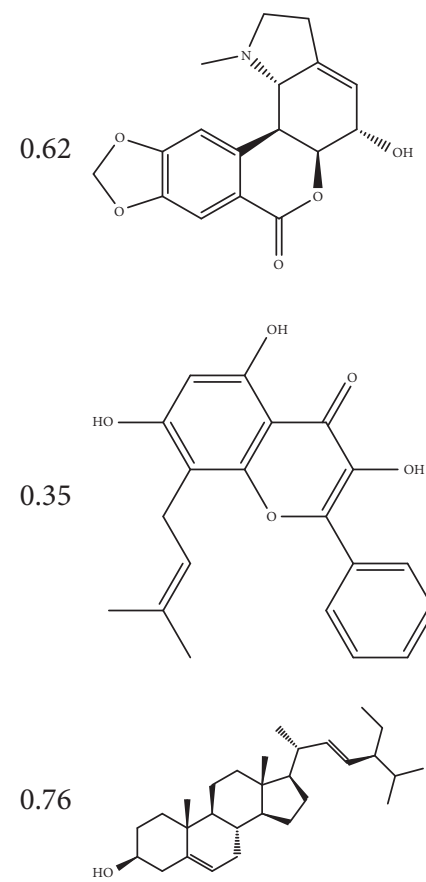

$61.07 \quad 0.35$

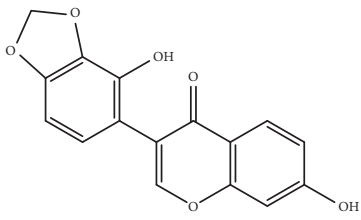


TABle 1: Continued.

\begin{tabular}{|c|c|c|c|c|}
\hline Herb & Molecule name & $\begin{array}{l}\text { OB } \\
(\%)\end{array}$ & $\mathrm{DL}$ & Structure \\
\hline $\mathrm{ZM}$ & Anemarsaponin F_qt & 60.06 & 0.79 & \\
\hline GC & Licocoumarone & 33.21 & 0.36 & \\
\hline $\mathrm{ZM}$ & Diosgenin & 80.88 & 0.81 & \\
\hline GC & Lupiwighteone & 51.64 & 0.37 & \\
\hline $\mathrm{ZM}$ & Mangiferolic acid & 36.16 & 0.84 & \\
\hline GC & Eurycarpin A & 43.28 & 0.37 & \\
\hline $\mathrm{ZM}$ & Anemarsaponin E_qt & 30.67 & 0.86 & \\
\hline GC & Dehydroglyasperins C & 53.82 & 0.37 & \\
\hline $\mathrm{ZM}$ & Timosaponin B III_qt & 35.26 & 0.87 & \\
\hline
\end{tabular}


TABle 1: Continued.

\begin{tabular}{|c|c|c|c|c|}
\hline Herb & Molecule name & $\begin{array}{l}\text { OB } \\
(\%)\end{array}$ & $\mathrm{DL}$ & Structure \\
\hline
\end{tabular}

GC

Gancaonin G

$\mathrm{ZM}$

GC

FL

ergosta-7, 22E-dien-3beta-ol

GC

FL

GC

FL

GC
Glyasperin C

Anemarsaponin C_qt

Gancaonin A

Poricoic acid B

Glyasperins M

Poricoic acid C

Sigmoidin-B

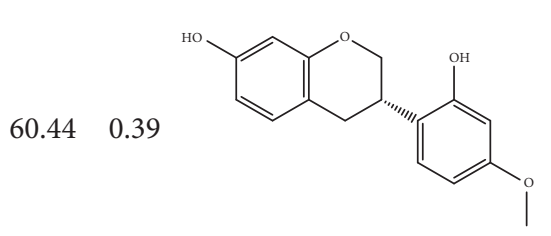

35.5

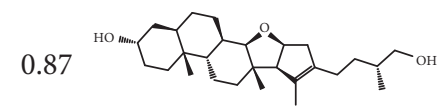

45.56

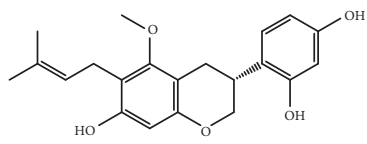

43.51

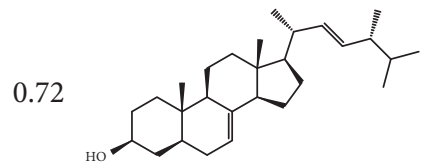

51.08

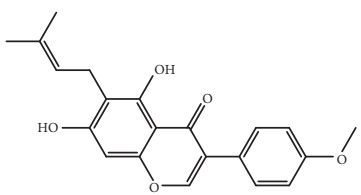

30.52

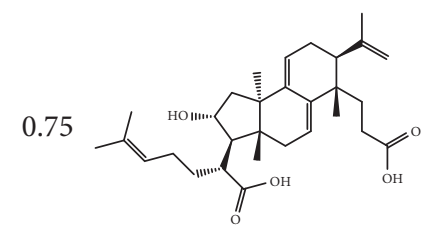

72.67

0.59<smiles>COc1cc(Br)cc2c1C(=O)[C@H](c1ccc3c(c1O)C=CC(C)(C)O3)CO2</smiles>

38.15

0.75<smiles>C=C(CCC(=O)O)CCC(C(=O)O)[C@H]1CCC2(C)C1=CC[C@@]1(C)C2=CC[C@@H]1C(=C)C</smiles>

4.88

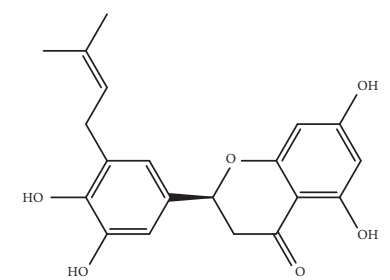


TABle 1: Continued.

Herb Molecule name

FL

GC

FL

GC

FL

GC
Poricoicacid A

Licoisoflavone

Cerevisterol

Isolicoflavonol

Trametenolic acid

Glyasperin B

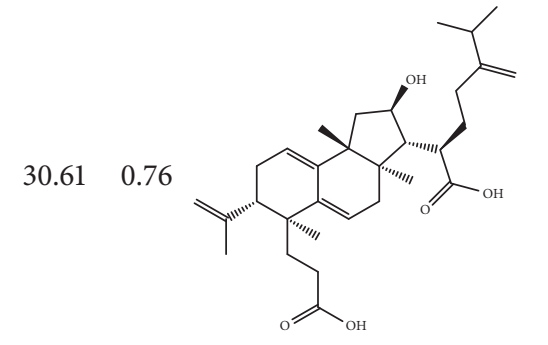

41.61

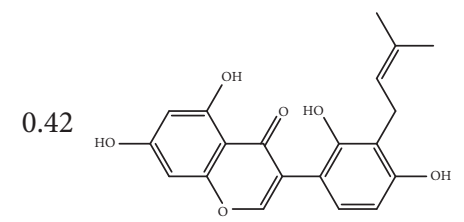

37.96<smiles>CC(C)C(C)/C=C/C(C)C1CCC2C3CC(C)C4(C)CC[C@@H](C)C[C@]4(C)C3CCC12C</smiles>

45.17<smiles>CC(C)=CCc1cc(-c2oc3cc(O)cc(O)c3c(=O)c2O)ccc1OC#N</smiles>

38.71

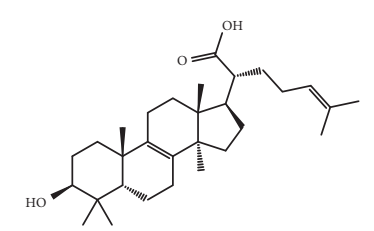

65.22

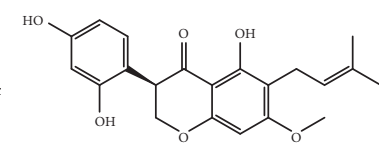


TABle 1: Continued.

\begin{tabular}{|c|c|c|c|c|}
\hline Herb & Molecule name & $\begin{array}{l}\text { OB } \\
(\%)\end{array}$ & $\mathrm{DL}$ & Structure \\
\hline
\end{tabular}

FL

$$
\text { 7, 9(11)-Dehydropachymic acid }
$$

GC

$$
\text { Glabrene }
$$

Ergosterol peroxide

GC

Phaseolinisoflavan

FL

GC

FL

GC

CX

GC
Pachymic acid

Gancaonin B

Dehydroeburicoic acid

Licoricone

Mandenol

Glycyrin

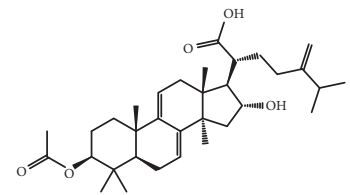

46.27

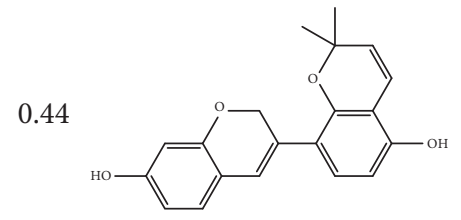

40.36

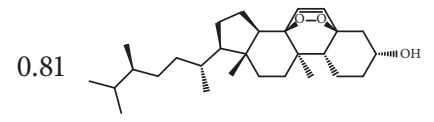

32.01

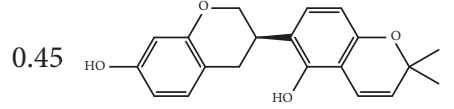

33.63

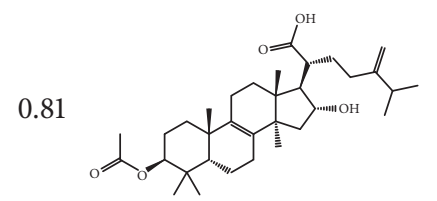

48.79

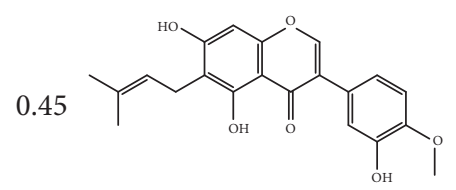

44.17

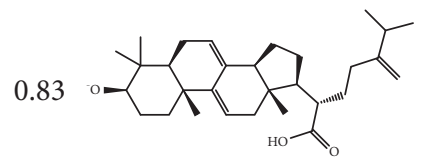

$63.58 \quad 0.47$<smiles>COC1=CC(OC)C(C2COCc3cc(Br)ccc3C2=O)C(OC)=C1CC=C(C)C</smiles>

42

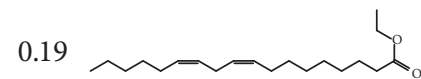

52.61

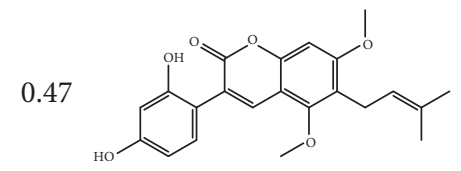


TABle 1: Continued.

\begin{tabular}{|c|c|c|c|c|}
\hline Herb & Molecule name & $\begin{array}{l}\text { OB } \\
(\%)\end{array}$ & $\mathrm{DL}$ & Structure \\
\hline CX & Senkyunone & 47.66 & 0.24 & \\
\hline GC & Glabridin & 53.25 & 0.47 & \\
\hline CX & Perlolyrine & 65.95 & 0.27 & \\
\hline GC & Licoagroisoflavone & 57.28 & 0.49 & \\
\hline CX & Myricanone & 40.6 & 0.51 & \\
\hline GC & Glabrone & 52.51 & 0.5 & \\
\hline CX & FA & 68.96 & 0.71 & \\
\hline GC & Kanzonols W & 50.48 & 0.52 & \\
\hline
\end{tabular}


Table 1: Continued.

\begin{tabular}{|c|c|c|c|c|}
\hline Herb & Molecule name & $\begin{array}{l}\text { OB } \\
(\%)\end{array}$ & $\mathrm{DL}$ & Structure \\
\hline
\end{tabular}

CX

GC

SZR

GC

SZR

GC

SZR

GC
Wallichilide

Inermine

(S)-Coclaurine

Glyasperin F

Zizyphusine

Licoisoflavanone

Jujuboside A_qt

Semilicoisoflavone B
42.31

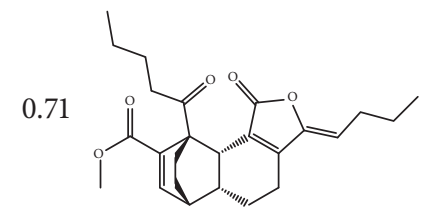

75.18

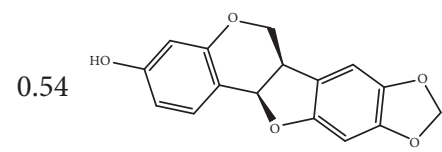

42.35

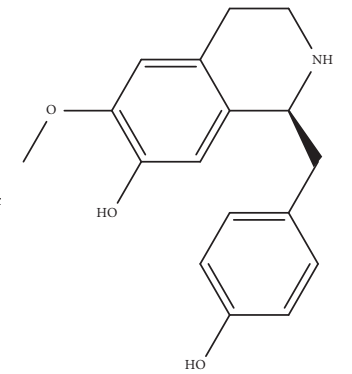

75.84<smiles>Cc1cc(O)cc2c1C(=O)[C@@H](c1ccc(O)c3c1OC(C)(C)C=C3)CO2</smiles>

41.53<smiles>COc1ccc2c(c1OC)-c1c(O)c(O)cc3c1[C@@H](C2)[N+](C)(C)CC3</smiles>

52.47

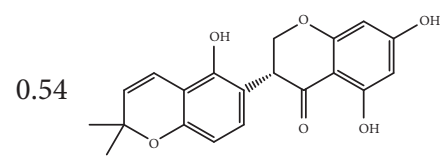

34.96

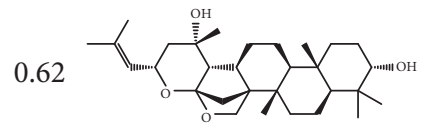

48.78

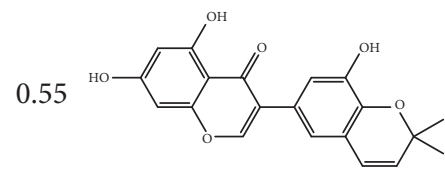


TABle 1: Continued.

\begin{tabular}{|c|c|c|c|c|}
\hline Herb & Molecule name & $\begin{array}{l}\text { OB } \\
(\%)\end{array}$ & $\mathrm{DL}$ & Structure \\
\hline SZR & Daucosterol & 36.91 & 0.75 & \\
\hline GC & Licoisoflavone B & 38.93 & 0.55 & \\
\hline SZR & Phytosterol & 36.91 & 0.75 & \\
\hline GC & $3^{\prime}$-Methoxyglabridin & 46.16 & 0.57 & \\
\hline SZR & Swertisin & 31.83 & 0.75 & \\
\hline GC & Licoagrocarpin & 58.81 & 0.58 & \\
\hline SZR & Ceanothic acid & 33.41 & 0.77 & \\
\hline GC & Phaseol & 78.77 & 0.58 & \\
\hline
\end{tabular}


TABle 1: Continued.

\begin{tabular}{lllll}
\hline Herb & Molecule name & OB \\
$(\%)$ & DL & Structure \\
\hline
\end{tabular}

SZR

GC

3,22-Dihydroxy-11-oxo-delta (12)-oleanene-27-alpha-methoxycarbonyl-29-oic acid

SZR,

GC

GC

1,3-Dihydroxy-8,9-Dimethoxy-6-Benzofurano[3,2-c]chromenone

GC

3-(3,4-Dihydroxyphenyl)-5,7-dihydroxy-8-(3-methylbut-2-enyl) chromone

GC

8-Prenylated eriodictyol

GC

5,7-Dihydroxy-3-(4-Methoxyphenyl)-8-(3-methylbut-2-enyl) chromone

GC

(2R)-7-Hydroxy-2-(4-hydroxyphenyl) chroman-4-one
67.28<smiles>CC(C)C[C@H](NC(=O)[C@@H](NC(=O)/C=C/c1ccccc1)C(C)C)C(=O)N/C=C/C1CCCCC1</smiles>

34.32

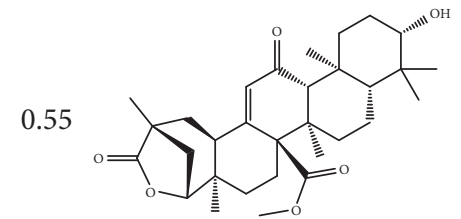

55.38

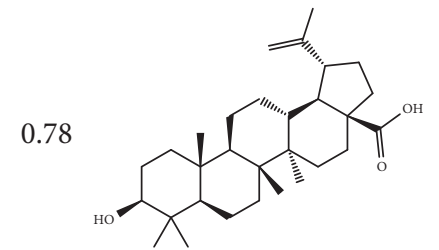

62.9<smiles></smiles>

66.37

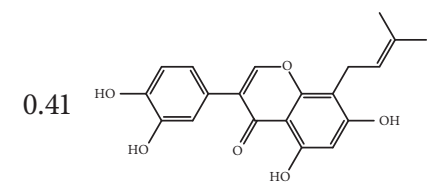

53.79

0.4<smiles>CC(C)CCc1c(O)cc(Cl)c2c1O[C@H](c1ccc(O)c(O)c1)CC2=O</smiles>

30.49

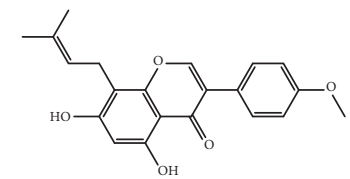

71.12<smiles>O=C1C[C@H](c2ccc(Br)cc2)Oc2cc([18O][Na])ccc21</smiles> 
TABle 1: Continued.

\begin{tabular}{|c|c|c|c|c|}
\hline Herb & Molecule name & $\begin{array}{l}\text { OB } \\
(\%)\end{array}$ & $\mathrm{DL}$ & Structure \\
\hline
\end{tabular}

GC 2-(3,4-Dihydroxyphenyl)-5,7-dihydroxy-6-(3-methylbut-2-enyl) chromone

(2S)-6-(2,4-Dihydroxyphenyl)-2-(2-hydroxypropan-2-yl)-4-methoxy-2,3-

GC dihydrofuro[3,2-g]chromen-7-one

GC

3-(2,4-Dihydroxyphenyl)-8-(1,1-dimethylprop-2-enyl)-7-hydroxy-5-methoxycoumarin

GC $7,2^{\prime}, 4^{\prime}$-Trihydroxy-5-methoxy-3-arylcoumarin

GC 1,3-Dihydroxy-9-methoxy-6-benzofurano[3,2-c]chromenone

GC

(E)-3-[3,4-Dihydroxy-5-(3-methylbut-2-enyl) phenyl]-1-(2,4-dihydroxyphenyl) prop-2-en-1-one

8-(6-Hydroxy-2-benzofuranyl)-2,2-dimethyl-5-chromenol

GC

(2S)-2-[4-Hydroxy-3-(3-methylbut-2-enyl) phenyl]-8,8-dimethyl-2,3dihydropyrano $[2,3-\mathrm{f}]$ chromen-4-one
44.15

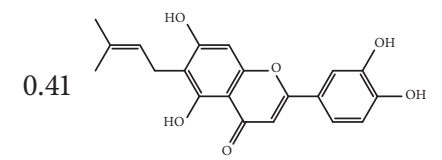

60.25

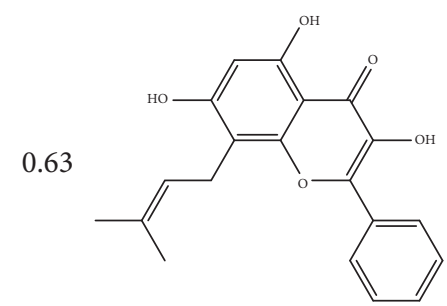

59.62

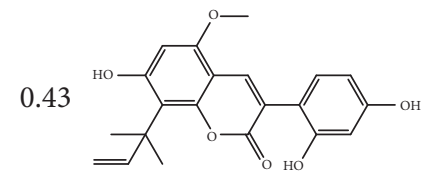

83.71<smiles>COc1cc(C)cc2oc(=O)c(-c3ccc(Cl)cc3O)cc12</smiles>

48.14<smiles></smiles>

46.27<smiles>CC(C)=CCc1cc(/C=C/C(=O)c2ccc(O)cc2O)cc(Br)c1O</smiles>

58.44<smiles>CC1(C)C=Cc2c(O)ccc(-c3cc4ccc([15OH])cc4o3)c2O1</smiles>

31.79

0.72

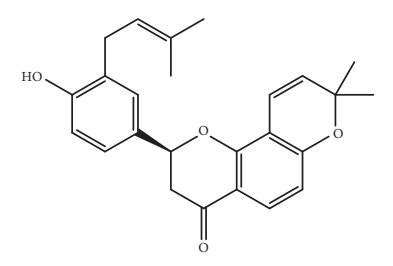


TABLE 1: Continued.

\begin{tabular}{|c|c|c|c|c|}
\hline Herb & Molecule name & $\begin{array}{l}\text { OB } \\
(\%)\end{array}$ & $\mathrm{DL}$ & Structure \\
\hline
\end{tabular}

$\mathrm{ZM}$

(Z)-3-(4-Hydroxy-3-methoxy-phenyl)-N-[2-(4-hydroxyphenyl) ethyl]acrylamide

(2R)-2-[(3S,5R,10S,13R,14R,16R,17R)-3,16-Dihydroxy-4,4,10,13,14-pentamethyl-

FL 2,3,5,6,12,15,16,17-octahydro-1H-cyclopenta[a]phenanthren-17-yl]-5-isopropylhex-5-enoic acid

(2R)-2-[(3S,5R,10S,13R,14R,16R,17R)-3,16-Dihydroxy-4,4,10,13,14-pentamethyl-

FL 2,3,5,6,12,15,16,17-octahydro-1H-cyclopenta[a]phenanthren-17-yl]-6-methylhept5-enoic acid

GC

7-Methoxy-2-methyl isoflavone

FL

3 $\beta$-Hydroxy-24-methylene-8-lanostene-21-oic acid

GC

(2S)-7-Hydroxy-2-(4-hydroxyphenyl)-8-(3-methylbut-2-enyl) chroman-4-one

GC

2-[(3R)-8,8-Dimethyl-3,4-dihydro-2H-pyrano[6,5-f]chromen-3-yl]-5methoxyphenol

GC

(E)-1-(2,4-Dihydroxyphenyl)-3-(2,2-dimethylchromen-6-yl) prop-2-en-1-one

GC

$$
3^{\prime} \text {-Hydroxy-4'-O-Methylglabridin }
$$

118.35<smiles>COc1cc(/C=C\C(=O)NCCc2ccc(O)cc2)ccc1O</smiles>

31.07

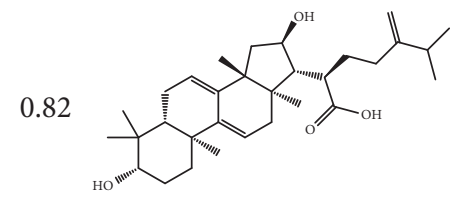

30.93

0.81<smiles>CC(C)=CCCC(C(=O)O)C1C(C)CC2C3=CCC4C(C)(C)C(C)CCC4(C)C3CCC2C1C(=O)C(C)C</smiles>

42.56

0.2<smiles>COc1ccc2c(=O)c(-c3ccccc3)c(C)oc2c1</smiles>

38.7<smiles>CC(=O)C(CCC(C)C(C)C)C1CCC2(C)C(C)CCC1=C1C2CCC2C1(C)CCC(C)C2(C)C</smiles>

36.57

0.32<smiles>CC(C)=CCc1c(O)ccc2c1O[C@H](c1ccc(O)cc1)CC2=O</smiles>

36.21

0.52

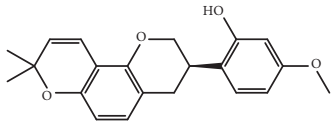

39.62<smiles>Cc1cc(O)ccc1C(=O)/C=C/C1=CC2C=CC(C)(C)OC2C=C1</smiles>

43.71

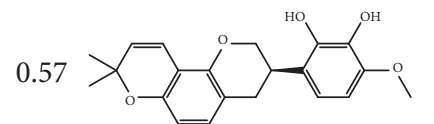


TABLE 1: Continued.

\begin{tabular}{|c|c|c|c|c|}
\hline Herb & Molecule name & $\begin{array}{l}\text { OB } \\
(\%)\end{array}$ & DL & Structure \\
\hline GC & 6-Prenylated eriodictyol & 39.22 & 0.41 & \\
\hline GC & 7-Acetoxy-2-methylisoflavone & 38.92 & 0.26 & \\
\hline FL & $\begin{array}{l}\text { (2R)-2-[(5R,10S,13R,14R,16R,17R)-16-Hydroxy-3-keto-4,4,10,13,14-pentamethyl- } \\
1,2,5,6,12,15,16,17 \text {-octahydrocyclopenta[a]phenanthren-17-yl]-5-isopropyl-hex-5- } \\
\text { enoic acid }\end{array}$ & 38.26 & 0.82 & \\
\hline
\end{tabular}

3.6. Docking Results of Active Compounds of SZRTand MAOB. AutoDock and PyMOL 2.7 software were used to dock 10 active ingredients such as quercetin, stigmasterol, coumaroyltyramine, glypallichalcone, and HMO with the key target MAOB. The docking results are shown in Table 3. The results show that 10 active ingredients could be docked with MAOB: coumaroyltyramine had the lowest binding energy with $\mathrm{MAOB},-9.6 \mathrm{kcal} / \mathrm{mol}$. Coumaroyltyramine formed hydrogen bonds with the amino acid residues LEU-164 and PHE-168 in MAOB (shown in Figure 6). HMO formed hydrogen bonds with the amino acid residues TYR-435 and TYR-326 in MAOB (shown in Figure 7). In addition, the binding energies of quercetin, isorhamnetin, and glypallichalcone to MAOB were also relatively low.

\section{Discussion}

In this study, network pharmacology and molecular docking were used to analyze the antianxiety mechanism of SZRT. The C-T network contains 79 nodes, 22 targets, and 57 active ingredients, forming 119 relationships. The C-T network analysis shows that the average degree of each target compound is 5.4, indicating the multitarget characteristics of SZRT. Quercetin exhibited the highest degree of interactions with multiple protein targets. Previous studies showed that quercetin can mitigate anxiety behavior in elevated plus maze and Morris water maze tests [14] and adriamycin-induced anxiety-like rat [15] by the normalization of HPA axis function [16] and regulate the GABAergic nervous system [17]. Glabridin is one of the main flavonoid compounds obtained from Glycyrrhiza glabra and exhibits antianxiety biological activity and acts as a potential GABAA receptor [18]. We speculate that the effect of SZRT on antianxiety might be regulated by the nervous system. Many potential targets are also linked with multiple compounds of different herbs, which might exhibit synergistic effects or additive effects of SZRT in the treatment of anxiety. NR3C1 is targeted by four active compounds (Anemarsaponin F_qt, Anemarsaponin C_qt, Timosaponin B III_qt, and wallichilide), which might provide additive effects to endocrine regulation.

Furthermore, the 22 predicted targets were analyzed. It was found that CCL2, CRP, CXCL8, IL10, IL1B, TNF, and TP53 were related to immunoregulation. DRD1, SLC6A3, MAOA, MAOB, HTR3A, ADRB2, and SLC6A2 belong to monoamine neurotransmitters and their receptors, and GABRA1 and GABRA2 belong to amino acid neurotransmitter receptors, participating in neurotransmitter regulation. Besides, SOD1 and PON1 are important oxidoreductases. NR3C1 is a glucocorticoid receptor. These results indicate that the effect of SZRT in the treatment of anxiety is associated with regulating neurotransmitters, inflammatory reaction, endocrine, and antioxidation, exhibiting a comprehensive regulatory effect.

For further evaluation, 16 pathways were mainly enriched in the multi-neurotransmitter regulation module and inflammatory regulation module. The pathways of multi-neurotransmitters could be approximately divided into two classes: (1) related to the synthesis, secretion, and recycling of neurotransmitters including dopaminergic synapse, serotonergic synapse, tryptophan metabolism, amphetamine addiction, and phenylalanine metabolism pathways and (2) related to the sedative or hypnotic effects such as morphine, addiction, and cocaine addiction. The pathways of neurotransmitter regulation were enriched in the targets of MAOA, MAOB, SLC6A3, and DRD1.

Anxiety is characterized by the disorder of neural factors. Many neurotransmitter substances such as 5-HT and DA are directly related to the occurrence of anxiety. The dopaminergic synapse and serotonergic synapse pathways play an 
(2S)-6-(2,4-dihydroxyphenyl)-2-(2-hydroxypropan-2-yl)-4-methoxy-2,3-dihydrofuro[3,2-g]chromen-7-one

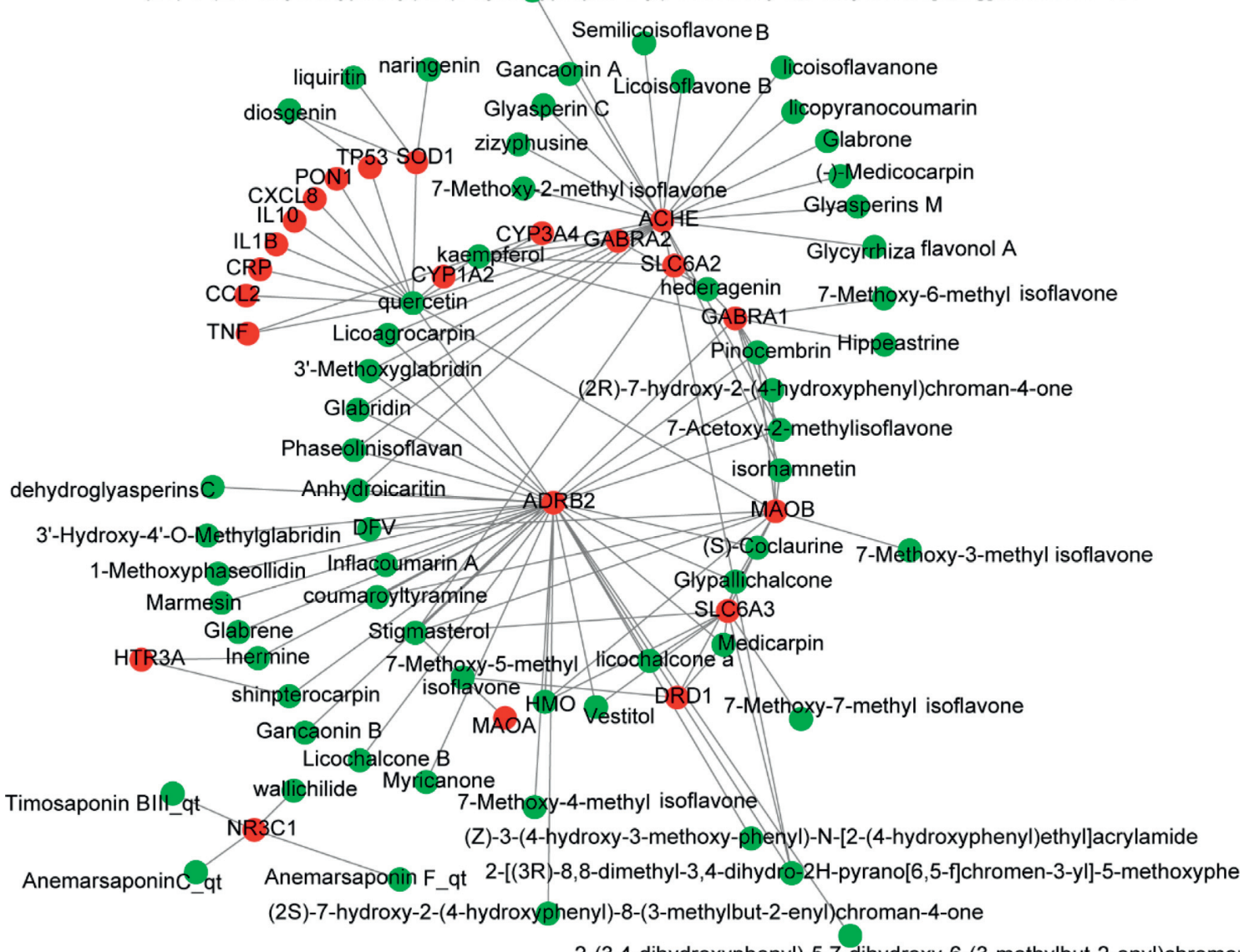

2-(3,4-dihydroxyphenyl)-5,7-dihydroxy-6-(3-methylbut-2-enyl)chromol

Figure 2: C-T network. A compound node and a protein node are linked if the protein is targeted by the corresponding compound. Node size is proportional to its degree. The green cycles indicates active compounds and red were targets.

TABLE 2: The related pathway of the antianxiety effect of SZRT.

\begin{tabular}{|c|c|c|c|}
\hline Term & ID & $P$ value & Input gene name \\
\hline Cytokine-cytokine receptor interaction & hsa04060 & $5.86 E-07$ & CCL2|CXCL8|IL1B|TNF|IL10 \\
\hline IL-17 signaling pathway & hsa04657 & $2.47 E-07$ & CCL2|CXCL8|IL1B|TNF \\
\hline NOD-like receptor signaling pathway & hsa04621 & $3.06 E-06$ & CCL2|CXCL8|IL1B|TNF \\
\hline NF-kappa B signaling pathway & hsa04064 & $2.60 E-05$ & CXCL8|IL1B|TNF \\
\hline Toll-like receptor signaling pathway & hsa04620 & $2.92 E-05$ & CXCL8|IL1B|TNF \\
\hline C-type lectin receptor signaling pathway & hsa04625 & $2.92 E-05$ & IL10|IL1B|TNF \\
\hline MAPK signaling pathway & hsa04010 & 0.0006 & IL1B|TNF|TP53 \\
\hline Neuroactive ligand-receptor interaction & hsa04080 & $1.15 E-06$ & ADRB2|GABRA2|GABRA1|NR3C1|DRD1 \\
\hline Amphetamine addiction & hsa05031 & $7.41 E-08$ & SLC6A3|MAOB|MAOA|DRD1 \\
\hline Dopaminergic synapse & hsa04728 & $9.32 E-07$ & SLC6A3|MAOB|MAOA|DRD1 \\
\hline Serotonergic synapse & hsa04726 & $3.91 E-05$ & MAOB|MAOA|HTR3A \\
\hline Morphine addiction & hsa05032 & $1.98 E-05$ & GABRA2|GABRA1|DRD1 \\
\hline Phenylalanine metabolism & hsa00360 & $5.09 E-05$ & MAOB|MAOA \\
\hline Tryptophan metabolism & hsa00380 & $2.13 E-06$ & МAOB|CYP1A2|MAOA \\
\hline Morphine addiction & hsa05032 & 0.0001 & GABRA2|GABRA1|DRD1 \\
\hline Cocaine addiction & hsa05030 & $2.12 E-08$ & SLC6A3|MAOB|MAOA|DRD1 \\
\hline
\end{tabular}

important role in the synthesis, secretion, and recycling of 5HT, DA, MAOA, MAOB, and SLC6A3 in dopaminergic synapse and serotonergic synapse pathway regulated by stigmasterol from ZM. Owing to its MAO enzyme-inhibiting properties, quercetin might provide the anticatatonic effect of L-dopa plus carbidopa treatment [19].
Anxiety is not only closely related to the nervous system, but it is also associated with immune function. Although the causal relationship between anxiety and immune abnormalities is not clear, an abnormal immune status can trigger anxiety, and immune dysfunction does not exist in patients with anxiety. Cytokines are involved in the regulation of mood and 


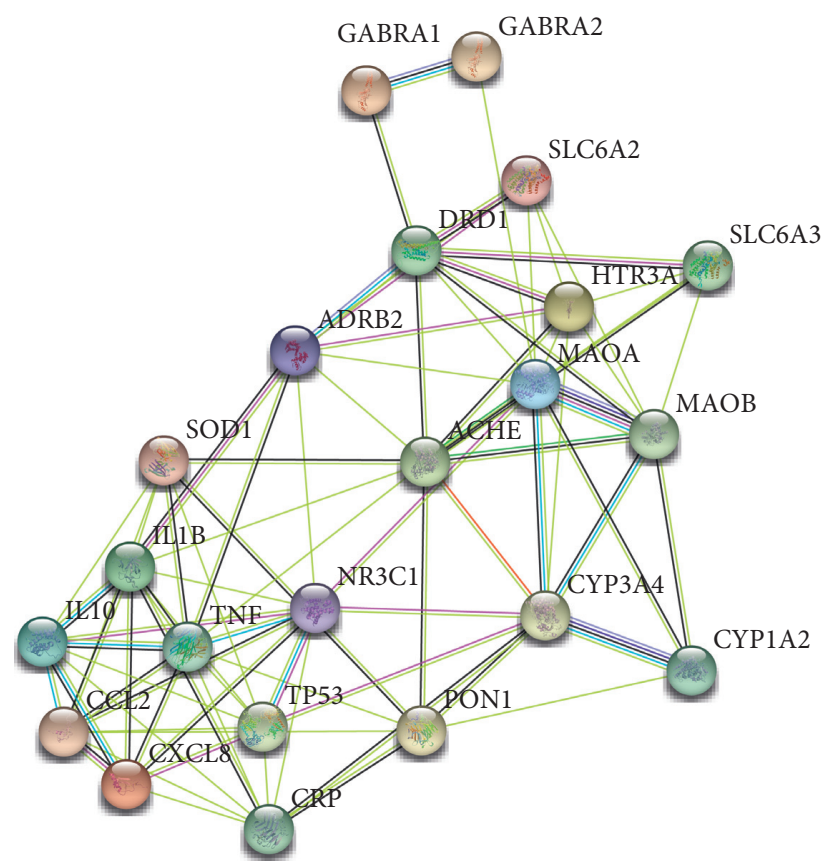

FIgURE 3: The PPI network for 22 targets.

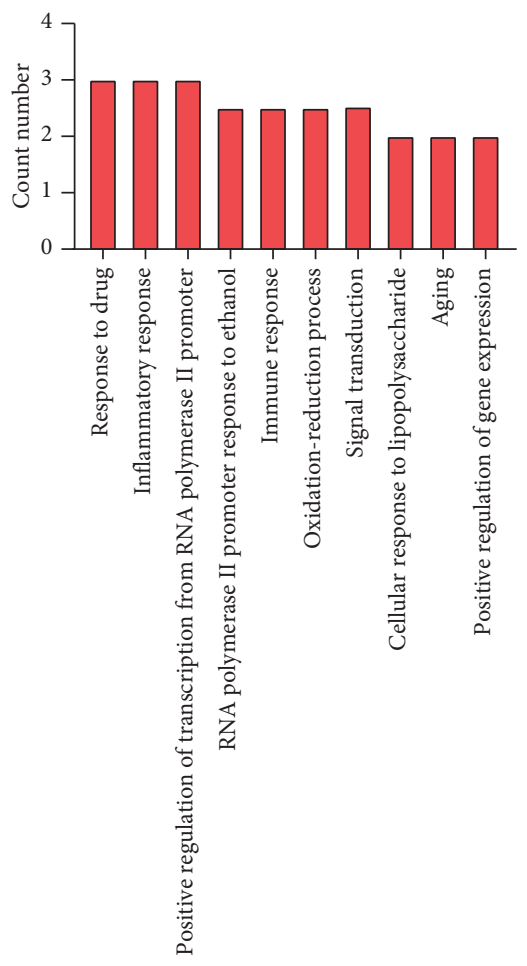

(a)

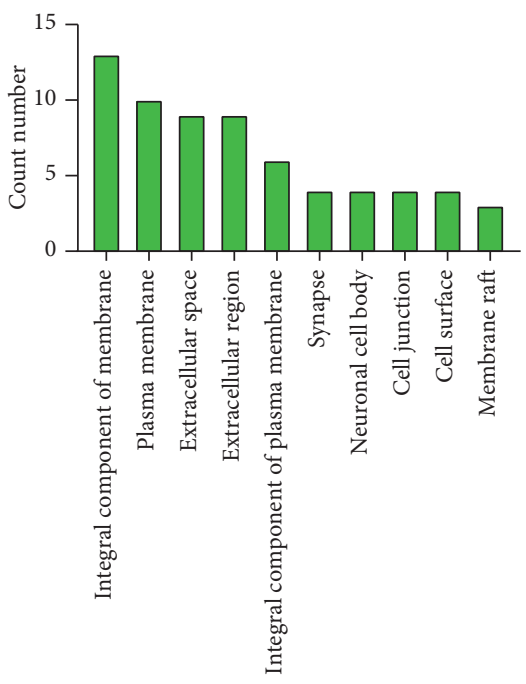

(b)

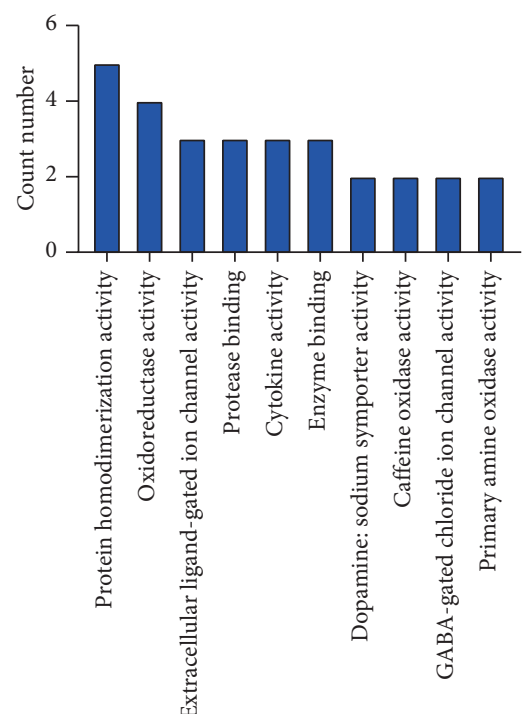

(c)

Figure 4: Gene Ontology (GO) analysis. (a) BF. (b) CC. (c) MF.

behavior and play an important role in mental disorders such as anxiety; for example, IL-4 knockout mice exhibit anxiety-like behavior [20]. Studies have shown that IL-1, IL-2, and TNF- $\alpha$ could induce anxiety in animals [21]; TNF- $\alpha$ could reduce the function of prefrontal cortex-limbic system, eventually causing anxiety and depression symptoms [22]. Zhang [23] also found that CRP, IL-1 $\alpha$, IL-6, IL-12, INF- $\gamma$, and GM-CSF levels were higher in patients with GAD, and they were positively 

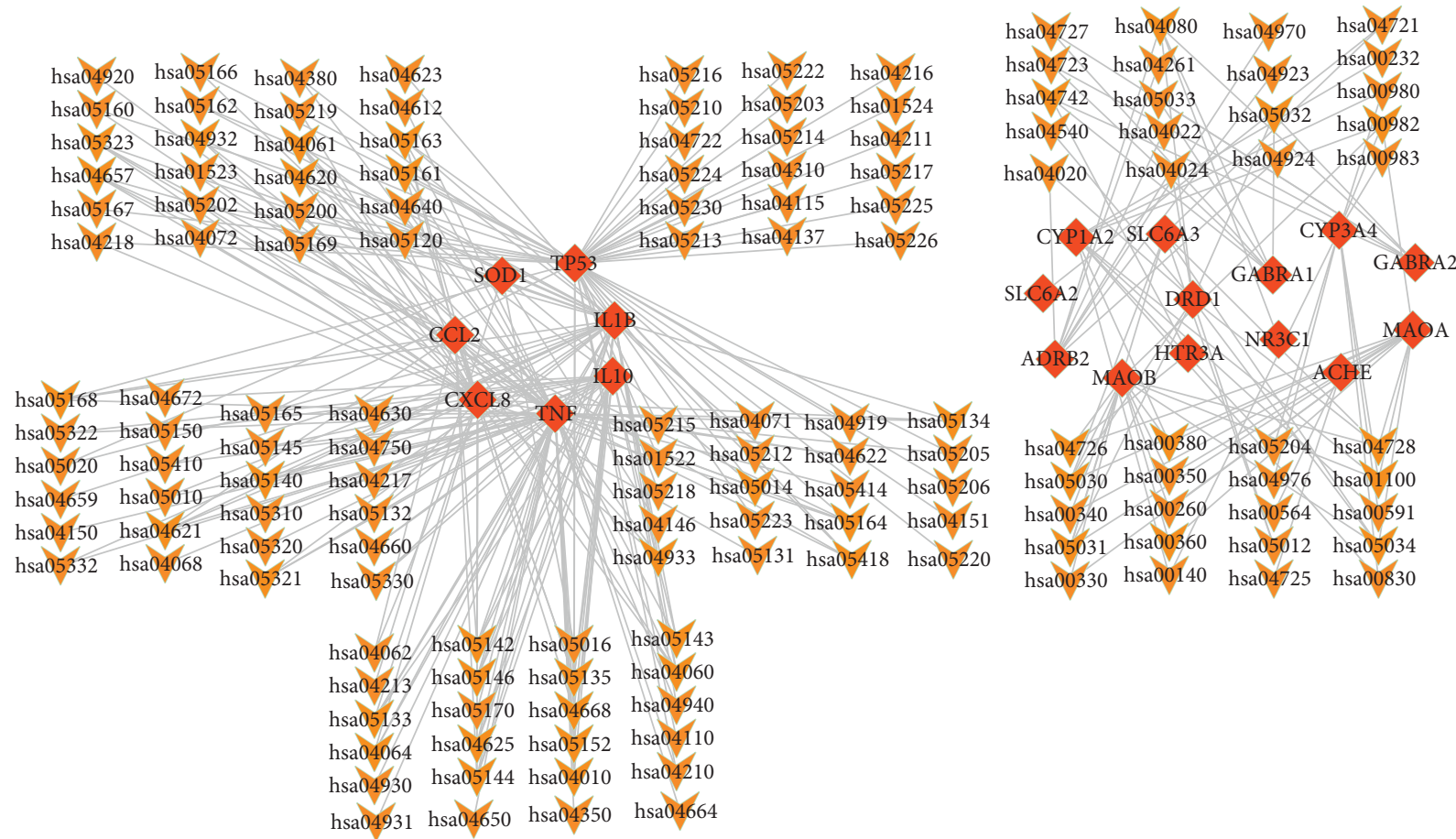

FIGURE 5: The targets-pathway network of SZRT for treating anxiety. The red diamond nodes represent genes and the yellow ones are pathways.

TABle 3: Predicted binding energies for active components of SZRT docked with MAOB.

\begin{tabular}{lcc}
\hline Targets $(\mathrm{V})$ & Active compounds & Binding energy (kcal/mol) \\
\hline 260 & Quercetin & -8.6 \\
260 & Isorhamnetin & -8.8 \\
260 & Stigmasterol & -3.3 \\
260 & coumaroyltyramine & -9.6 \\
260 & (S)-Coclaurine & -7.5 \\
260 & DFV & -8.7 \\
260 & Pinocembrin & -6.3 \\
260 & 7-Methoxy-2-methyl isoflavone & -7.6 \\
260 & Glypallichalcone & -8.6 \\
260 & HMO & -8.7 \\
\hline
\end{tabular}

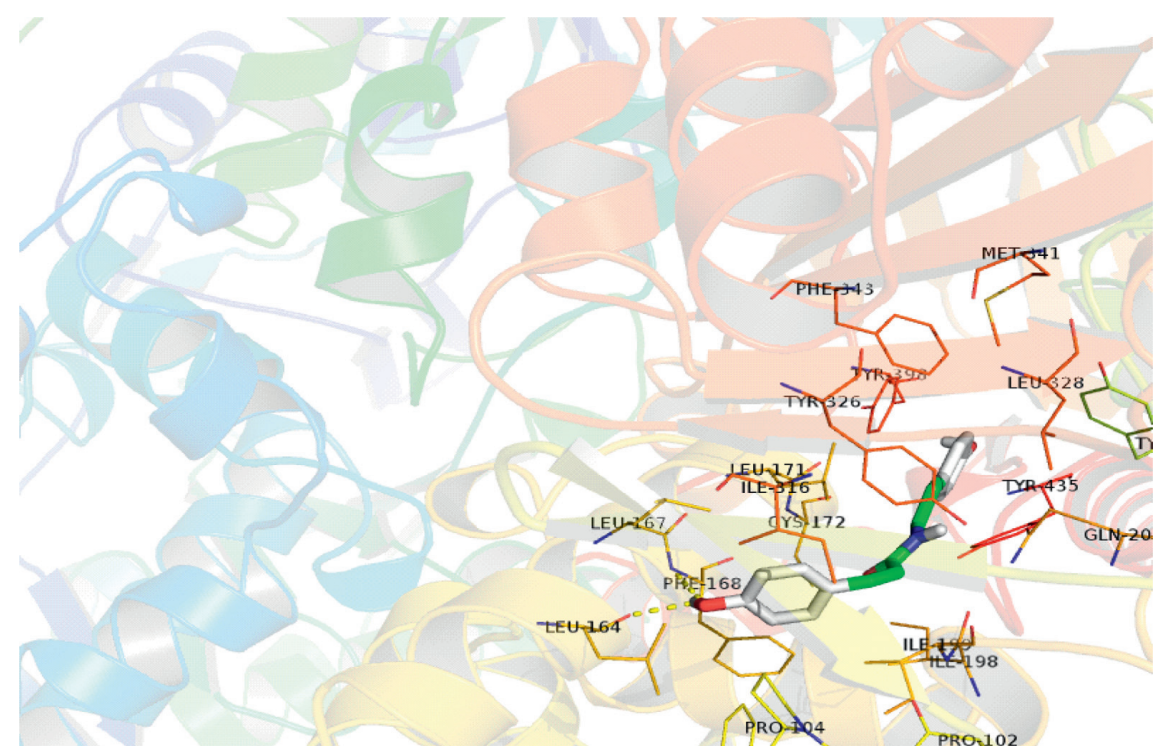

FIGURE 6: The molecular docking result of coumaroyltyramine with MAOB. 


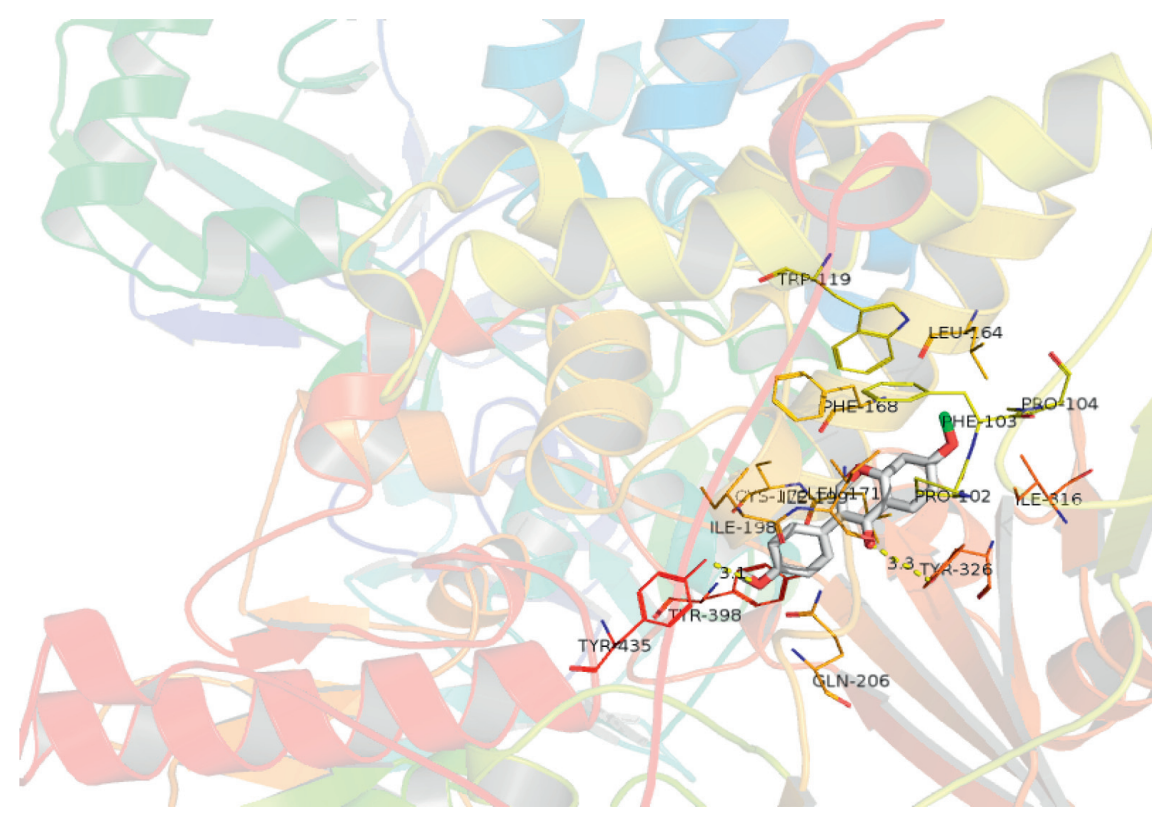

Figure 7: The molecular docking result of HMO with MAOB.

correlated with the severity of anxiety disorder. Therefore, it is speculated that an inflammatory response activation system might be involved in the regulation of anxiety.

In this study, CCL2, CXCL8, IL1B, TNF, and IL10 were found to have enriched cytokine-cytokine receptor interaction, IL-17 signaling pathway, NOD-like receptor signaling pathway, and NF-kappa B signaling. Previous studies have shown that some cytokines and chemokines such as IL1B can impact the activity of monoamine neurotransmitters and cause anxiety-like behavior [24]. SZRT showed lower serum TNF- $\alpha$ and IL-2 levels in EMP model rats [25]. These results indicate that the mechanism of SZRT anxiolytic efficacy might be related to immunoregulation.

The pathogenesis of anxiety is complex. More and more researches have been made on the relationship between oxidative stress and anxiety. The research of Hovatta showed that the antioxidation defense mechanism was closely related to anxiety [26]. Clinical research found that serum superoxide dismutase (SOD) in patients with GAD was positively correlated with total anxiety score; MDA was negatively related to it [27]. And improving of oxidative stress might be one of the antianxiety mechanisms [28, 29]. The same results were also shown in various anxiety model animals. The contents of catalase (CAT) and SOD in brain tissue and serum of anxiety animals were significantly reduced [30, 31]. Antioxidant therapy could alleviate the anxiety symptoms of model animals. Liquiritin, naringenin, diosgenin, and quercetin are potential active components in SZRT and mapped to anxiety-related targets of SOD1. Liquiritin, naringenin, and quercetin are flavonoid antioxidant and diosgenin isolated from ZM, which all have a remarkable antioxidant effect [32-34] and enhanced SOD activity in many animal models. It had also been reported that quercetin and naringenin also alleviate anxiety-like behaviors and regulated oxidative signs $[35,36]$. These indicated that the anxiolytic effects of SZRT may be related to antioxidant, and the ingredients of liquiritin, naringenin, diosgenin, and quercetin are the key antioxidants.

Further molecular docking analysis showed that the active ingredients predicted by the pharmacophore target can all bind to the related target protein, and coumaroyltyramine has a strong binding activity to monoamine oxidase MAOB. This study initially revealed the potential active ingredients of SZRT anxiolytic activity and its possible mechanism of action, providing a basis for further research on the material basis of SZRT anxiolytic efficacy and development of antianxiety components of TCM.

This study utilized network pharmacology and molecular docking methods based on the TCMSP database, GeneCards database, and KEGG database to study the potential material basis and antianxiety mechanism of SZRT. By analyzing the predicted targets and pathways, it was found that 57 potential active ingredients of SZRT correspond to 22 anxiety targets. These active ingredients and targets may help to further verify the antianxiety mechanism of SZRT. The C-T network and PPI network analyses showed that the core active ingredients of SZRT such as sitosterol, kaempferol, quercetin, and 7-methoxy-2methyl isoflavone might act on targets such as MAOA, MAOB, IL1B, TNF, NR3CI, and HTR3A, affecting the neurotransmitter regulation and immunoregulation pathways to produce anxiolytic effects. In this study, network pharmacology and molecular docking methods were used to predict the anxiolytic effects of SZRT and provide a theoretical basis for its further development and application. However, the specific mechanism and material basis should be confirmed in vivo and in vitro.

\section{Data Availability}

The data to support the findings of this study are available from the corresponding author upon request. 


\section{Disclosure}

Xiaocong Xu and Bingbing Gao are the co-first authors.

\section{Conflicts of Interest}

The authors declare that they have no conflicts of interest regarding the publication of this paper.

\section{Authors' Contributions}

Shanshan Lei conceived and designed the experiments. Bingbing Gao, Xiaocong $\mathrm{Xu}$, and Xiongying Li contributed significantly to analysis and manuscript preparation. Xiaocong Xu and Bingbing Gao contributed equally to this work.

\section{Acknowledgments}

This study was supported by the National Science Foundation of China (no. 81803760).

\section{References}

[1] Y.Z. Hua, "Lancet: with so many drugs for generalized anxiety disorder, which one should we choose?" Chin J Stroke, vol. 14, no. 4, pp. 357-358, 2019.

[2] J. Diemer, N. Vennewald, K. Domschke, and P. Zwanzger, "Therapy-refractory panic: current research areas as possible perspectives in the treatment of anxiety," European Archives of Psychiatry and Clinical Neuroscience, vol. 260, no. S2, pp. 127-131, 2010.

[3] M. G. Craske and M. B. Stein, “Anxiety," The Lancet, vol. 388, no. 10063, pp. 3048-3059, 2016.

[4] L. J. Yan and W. Yin, "Clinical analysis of Suanzaoren decoction in the treatment of generalized anxiety disorder," Journal of Shandong University of TCM no., vol. 06, pp. 39-40, 2004.

[5] L. L. Hu, X. Zhang, W. J. Liu et al., "Combination with Zhi zi chi tang as a treatment protocol for insomniacs with anxiety: a randomized parallel-controlled trial," Evidence-Based Complementray Alternative Medicine, vol. 7, Article ID 913252, 2015.

[6] X. Ming, W. Xin, W. S. Yong et al., "Study on the anti anxiety effect of Suanzaoren Decoction and its material basis, in China," Journal of Traditional Chinese Medicine, pp. 156-159, 2004.

[7] R. C. Lei, D. Y. Xia, and C. Ying, "Effects of Semen Ziziphi Spinosae on the anxiety behavior of the yin deficiency mice," Journal of Chinese Medicinal Materials, vol. 31, no. 11, pp. 1703-1705, 2008.

[8] C. Ying, L. Ju, D. Y. Xia, F. Jing, J. H. Jun, and S. L. Zhi, "Effects of Rhizoma Anemarrhenae on the anxiety behavior of then n deficiency mice," Chinese Journal of Behavioral Medical Science, vol. 17, no. 6, pp. 520-522, 2008.

[9] W. S. Yong, X. Ming, and W. Xin, "Correlation between component compatibilities of suanzaoren decoction and their effects of anti-anxiety," Chinese Journal of Experimental Traditional Medical Formulae, vol. 16, no. 3, pp. 105-108, 2010.

[10] Y.-H. Chen, S.-S. Lei, B. Li et al., "Systematic understanding of the mechanisms of flos chrysanthemi indici-mediated effects on hypertension via computational target fishing,"
Combinatorial Chemistry and High Throughput Screening, vol. 23, no. 2, pp. 92-110, 2020.

[11] H. Bao, H. Guo, Z. Feng, and X. Li, "Deciphering the underlying mechanism of Xianlinggubao capsule against osteoporosis by network pharmacology," BMC Complementary Medicine and Therapies, vol. 20, no. 1, p. 208, 2020.

[12] H. E. Lee, S. J. Jeon, B. Ryu et al., "Swertisin, a C-glucosylflavone, ameliorates scopolamine-induced memory impairment in mice with its adenosine A1 receptor antagonistic property," Behavioural Brain Research, vol. 306, pp. 137-145, 2016.

[13] R. M. Hussein, W. R. Mohamed, and H. A. Omar, “A neuroprotective role of kaempferol against chlorpyrifos-induced oxidative stress and memory deficits in rats via GSK3 $\beta$-Nrf2 signaling pathway," Pesticide Biochemistry and Physiology, vol. 152, pp. 29-37, 2018.

[14] A. Priprem, J. Watanatorn, S. Sutthiparinyanont, W. Phachonpai, and S. Muchimapura, "Anxiety and cognitive effects of quercetin liposomes in rats," Nanomedicine: Nanotechnology, Biology and Medicine, vol. 4, no. 1, pp. 70-78, 2008.

[15] S. Merzoug, M. L. Toumi, and A. Tahraoui, "Quercetin mitigates Adriamycin-induced anxiety- and depression-like behaviors, immune dysfunction, and brain oxidative stress in rats," Naunyn-Schmiedeberg's Archives of Pharmacology, vol. 387, no. 10, pp. 921-933, 2014.

[16] M. Kosari-Nasab, G. Shokouhi, A. Ghorbanihaghjo, M. Mesgari-Abbasi, and A.-A. Salari, "Quercetin mitigates anxiety-like behavior and normalizes hypothalamus-pituitary-adrenal axis function in a mouse model of mild traumatic brain injury," Behavioural Pharmacology, vol. 30, no. 2-3, pp. 282-289, 2019.

[17] J. J. Wook and L. S. Heon, "Anxiolytic effects of quercetin: involvement of GABAergic system," Journal of Life Science, vol. 24, no. 3, pp. 290-296, 2014.

[18] K. M. Hoffmann, L. Beltrán, P. M. Ziemba, H. Hatt, and G. Gisselmann, "Potentiating effect of glabridin from Glycyrrhiza glabra on GABA A receptors," Biochemistry and Biophysics Reports, vol. 6, pp. 197-202, 2016.

[19] A. Singh, P. S. Naidu, and S. K. Kulkarni, "Quercetin potentiates L-dopa reversal of drug-induced catalepsy in rats: possible COMT/MAO inhibition," Pharmacology, vol. 68, no. 2, pp. 81-88, 2003.

[20] M. L. Moon, J. J. Joesting, N. A. Blevins et al., "IL-4 knock out mice display anxiety-like behavior," Behavior Genetics, vol. 45, no. 4, pp. 451-460, 2015.

[21] C. R. Pawlak, R. K. Schwarting, and A. Bauhofer, "Cytokine mRNA levels in brain and peripheral tissues of the rat: relationships with plus-maze behavior, Brain Research," Molecular Brain Research, vol. 137, no. 1-2, pp. 159-165, 2005.

[22] B. E. Leonard and A. Myint, "The psychoneuroimmunology of depression," Human Psychopharmacology Clinical \& Experimental, vol. 24, no. 3, pp. 165-175, 2010.

[23] Y. H. Zhang, "Serum inflammatory factor level and significance in patients with generalized anxiety disorder," Journal of International Psychiatry, vol. 46, no. 3, pp. 428-430, 2019.

[24] J. W. Koo and R. S. Duman, "Interleukin-1 receptor null mutant mice show decreased anxiety-like behavior and enhanced fear memory," Neuroscience Letters, vol. 456, no. 1, pp. 39-43, 2009.

[25] W. S. Yong, X. Ming, and W. Xin, "Effects of Suanzaoren Decoction on neuropeptide $\mathrm{Y}$ and cytokines in rats with elevated cross maze," Chinese Traditional Patent Medicine, vol. 33, no. 1, pp. 142-145, 2011. 
[26] O. Coskun, M. Kanter, A. Korkmaz, and S. Oter, "Quercetin, a flavonoid antioxidant, prevents and protects streptozotocininduced oxidative stress and beta-cell damage in rat pancreas," Pharmacological Research, vol. 51, no. 2, pp. 117-123, 2005.

[27] Y. Cui, Y. Che, and H. Wang, "Bergamot essential oil attenuate aluminum-induced anxiety-like behavior through antioxidation, anti-inflammatory and GABA regulation in rats," Food and Chemical Toxicology, vol. 145, Article ID 111766, 2020.

[28] H. J. Heo, D.-O. Kim, S. C. Shin, M. J. Kim, B. G. Kim, and D.-H. Shin, "Effect of antioxidant flavanone, naringenin, from citrus junos on neuroprotection," Journal of Agricultural and Food Chemistry, vol. 52, no. 6, pp. 1520-1525, 2004.

[29] I. Hovatta, J. Juhila, and J. Donner, "Oxidative stress in anxiety and comorbid disorders," Neuroscience Research, vol. 68 , no. 4 , pp. $261-275,2010$.

[30] z. x. hua and x. lu, "Changes of Plasma SOD activity and MDA content in patients with depression and anxiety," Journal Of Convalescence and Rehabilitation, vol. 12, no. 4, pp. 157-158, 1997.

[31] C. J. MEI, G. Y. LAN, Z. X. RUI et al., "Prior exercise impairments anxiety-like behavior and increase in amygdala antioxidant capacity and BDNF expression in sleep deprived rats," Journal of Shenyang Sport University, vol. 35, no. 3, pp. 79-84, 2016.

[32] A. S. Olugbemide, B. Ben-Azu, A. G. Bakre, A. M. Ajayi, O. Femi-Akinlosotu, and S. Umukoro, "Naringenin improves depressive- and anxiety-like behaviors in mice exposed to repeated hypoxic stress through modulation of oxido-inflammatory mediators and NF-kB/BDNF expressions," Brain Research Bulletin, vol. 169, pp. 214-227, 2021.

[33] Y.-X. Sun, Y. Tang, A.-L. Wu et al., "Neuroprotective effect of liquiritin against focal cerebral ischemia/reperfusion in mice via its antioxidant and antiapoptosis properties," Journal of Asian Natural Products Research, vol. 12, no. 12, pp. 10511060, 2010.

[34] M. L. Toumi, S. Merzoug, B. Baudin, and A. Tahraoui, "Quercetin alleviates predator stress-induced anxiety-like and brain oxidative signs in pregnant rats and immune count disturbance in their offspring," Pharmacology Biochemistry and Behavior, vol. 107, pp. 1-10, 2013.

[35] C. Vollert, M. Zagaar, I. Hovatta et al., "Exercise prevents sleep deprivation-associated anxiety-like behavior in rats: potential role of oxidative stress mechanisms," Behavioural Brain Research, vol. 224, no. 2, pp. 233-240, 2011.

[36] P. Y. Yuan, W. Y. Yan, and L. Yu, "Effect of Jiuwei Zhenxin Granule on oxidative stress in patients with generalized anxiety disorde," Modern Journal of Integrated Traditional Chinese and Western Medicine, vol. 25, no. 36, pp. 4044-4046, 2016. 